\title{
La importancia de la estadística y el aprendizaje colaborativo en los institutos superiores tecnológicos de la provincia de Bolívar, Ecuador
}

\author{
The importance of statistics and collaborative learning in higher \\ technological institutes in the province of Bolívar, Ecuador \\ A importância da estatística e da aprendizagem colaborativa em institutos \\ de alta tecnologia na província de Bolívar, Equador \\ WELLINGTON ISAAC MALIZA CRUZ ${ }^{1}$ \\ ÁNGEL ISRAEL VELOZ GONZÁLEZ² \\ MIGUEL ÁNGEL TISALEMA SISA ${ }^{3}$
}

\begin{abstract}
RESUMEN
Este trabajo tiene como propósito analizar la importancia del aprendizaje colaborativo para la enseñanza de la estadística y cómo esta metodología transforma positivamente la manera de enseñar esta disciplina. La metodología utilizada fue de tipo exploratorio, bibliográfica de campo y cuantitativa, las cuales permitieron establecer un análisis con relación a la estrategia metodológica que los docentes utilizan en la construcción del conocimiento en el instituto superior donde se desarrolló el estudio. En la parte bibliográfica permitió la exhaustiva revisión del tema para conocer el estado de la cuestión, mediante la búsqueda, recopilación, estimación, crítica e información teórica relevante. En la parte cuantitativa la investigación presenta información tabulada que permiten ilustrar con objetividad los resultados alcanzados. Entre ellos, se destaca la percepción que docentes y educandos tienen acerca de cómo el aprendizaje colaborativo impacta en lo académico, lo social y lo psicológico. Asimismo, en qué sentido esta modalidad ayuda a fomentar la responsabilidad, la comunicación, el trabajo en equipo y el proceso de grupo. De igual manera, hasta qué punto este tipo de aprendizaje fomenta las relaciones interpersonales, eleva la autoestima y el sentido de pertenencia de los beneficiarios.
\end{abstract}

Palabras clave: Estadística; proceso de aprendizaje; contexto de aprendizaje; rendimiento académico; integración social.

\begin{abstract}
The purpose of this work is to analyze the importance of collaborative learning for the teaching of statistics and how this methodology positively transforms the way of teaching this discipline. The methodology used was of an exploratory, bibliographic field and quantitative type, which allowed establishing an analysis in relation to the methodological strategy that teachers use in the construction of knowledge in the higher institute where the study


was carried out. In the bibliographical part, it allowed the exhaustive revision of the topic to know the state of the question; through the search, compilation, estimation, criticism and relevant theoretical information. In the quantitative part, the research presents tabulated information that allows objectively illustrating the results achieved. Among them, the perception that teachers and students have about how collaborative learning impacts academically, socially and psychologically stands out. Likewise, in what sense does this modality help foster responsibility, communication, teamwork and the group process. Similarly, to what extent this type of learning fosters interpersonal relationships, increases the beneficiaries' self-esteem and sense of belonging.

Keywords: Statistics; learning process; learning context; academic performance; social integration.

\section{RESUMO}

O objetivo deste trabalho é analisar a importância do aprendizado colaborativo para o ensino de estatística e como essa metodologia transforma positivamente a maneira de ensinar essa disciplina. A metodologia utilizada foi de campo exploratório, bibliográfico e quantitativo, o que permitiu estabelecer uma análise em relação à estratégia metodológica utilizada pelos professores na construção do conhecimento no instituto superior em que o estudo foi realizado. Na parte bibliográfica, permitiu a revisão exaustiva do tópico para conhecer o estado da questão, através da busca, compilação, estimação, crítica e informação teórica relevante. Na parte quantitativa, a pesquisa apresenta informações tabuladas que permitem ilustrar objetivamente os resultados alcançados. Entre eles, destaca-se a percepção que professores e alunos têm sobre o impacto do aprendizado colaborativo em termos acadêmicos, sociais e psicológicos. Da mesma forma, em que sentido essa modalidade ajuda a promover a responsabilidade, a comunicação, o trabalho em equipe e o processo do grupo. Da mesma forma, em que medida esse tipo de aprendizado promove relacionamentos interpessoais, aumenta a auto-estima e o sentimento de pertencimento dos beneficiários.

Palavras-chave: Estatística; processo de aprendizagem; contexto de aprendizagem; rendimento acadêmico; integração social.

\section{INTRODUCCIÓN}

La estadística impacta prácticamente todos los aspectos de nuestra vida, porque a partir de todas nuestras actividades es posible recopilar datos que, después de ser analizados, nos permiten tomar decisiones. A fines del siglo XX emerge el concepto de aprendizaje colaborativo, el constructivismo a cobijado a este enfoque en el que se manifiesta que todo aprendizaje es social y mediado, los primeros estudios sobre la necesidad de desarrollar este tipo de proceso, se remontan a los años cuarenta, se replantea en la forma de enseñar mediante la interacción humana, con el propósito de preparar al individuo en valores democráticos y trabajo en equipo. 
En este contexto, en el Instituto Superior Tecnológico Guaranda, con la finalidad de promover el trabajo en equipo y el aprendizaje se consolide a través de pares, fomenta el aprendizaje colaborativo utilizando el aprendizaje de la estadística con la intencionalidad que los estudiantes se desarrollen socialmente al actuar de forma democrática y las actividades que se realizan en los procesos de enseñanza se ejecuten en grupo, con la orientación significativa del docente quien mediante sus instrucciones designa roles para conseguir los objetivos y los conocimientos necesarios para la consecución del perfil de egreso.

En este sentido, se ha adoptado como método innovador de enseñanza de la estadística utilizando el aprendizaje colaborativo, el cual, según el documento electrónico publicado por el Tecnológico Monterrey (s.f.) es "una técnica didáctica que promueve el aprendizaje centrado en el alumno basando el trabajo en pequeños grupos", es decir, el éxito de este método consiste la organización de pequeños grupos para la realización de trabajos en conjunto, la responsabilidad de las actividades áulicas para la construcción de los aprendizajes recae sobre los grupos de trabajo y no tanto sobre el docente, como lo afirma Barkley, Major \& Cross (2008), "Los estudiantes trabajan por parejas o en pequeños grupos para lograr unos objetivos de aprendizaje comunes. Es aprender mediante el trabajo engrupo, en vez de hacerlo trabajando solo".

\section{Beneficios del aprendizaje colaborativo}

Para Roberts (2005) los principales beneficios que el aprendizaje colaborativo aporta a los estudiantes se describen de la siguiente manera:

- Académicos puesto que enfatiza en la participación y construcción activa de conocimiento, promoviendo habilidades de alto orden de pensamiento e incrementando los resultados de la clase.

- Sociales, teniendo en cuenta que fomenta la creación de una atmósfera positiva para el aprendizaje a la vez que permite desarrollar un sistema de apoyo social para los estudiantes, fomentando la comprensión y enseñanza entre ellos;

- Psicológicos, ya que desarrolla actitudes positivas hacia los profesores y puede incrementar la autoestima de los estudiantes.

Las aplicaciones de la estadística en la vida diaria se reflejan en decisiones tan triviales como abordar el transporte público fuera de horas pico, o no acudir al supermercado los días de cobro de salario.

\section{Las cinco aplicaciones principales de la estadística en la vida diaria}

1. En el campo laboral: la estadística suele ser empleada en distintas áreas del campo laboral. La planeación estratégica de una organización se apoya fundamentalmente en estudios de pronósticos y de presupuestos. Los mecanismos de control, a cargo de los departamentos de cumplimiento, se aplican en función de los resultados históricos obtenidos a partir de estudios estadísticos.

2. En la preferencia por ciertas marcas: los consumidores de bienes y servicios generalmente manifiestan preferencia por ciertas marcas del mercado. Esta tendencia es producto del pensamiento estadístico según el cual la durabilidad, la calidad y el nivel de satisfacción es representado en mayor medida por estas marcas. 
3. En las finanzas personales: la planificación financiera de una persona es el vívido ejemplo de la aplicación de la estadística en la vida diaria. La relación entre los ingresos y los egresos determinan la situación actual de la persona. Estos datos sirven de fundamento histórico para la planificación de compromisos sucesivos que requieren el trazado de ciertas estrategias para ser logrados.

4. En los deportes: los récords de los deportistas son elaborados en función de su desempeño por cantidad de juegos o partidos en los cuales hayan participado. En el caso de los jugadores de béisbol, su desempeño se mide por su porcentaje de bateos, carreras anotadas y bases robadas. En el caso de los futbolistas delanteros, el patrón de referencia es la cantidad de goles por partidos jugados. Los datos estadísticos recabados constituyen elementos objetivos que conllevan a la mejor utilización de los recursos y de los entrenamientos. Es así como se logra que los atletas alcancen su máximo rendimiento.

5. En las ventas: el mundo de las ventas es planificado en función a análisis pormenorizados sobre las necesidades de los consumidores, sus gustos y sus preferencias. La medición de la calidad del servicio, del nivel de satisfacción de los clientes y las propias estrategias de ventas es determinada por la aplicación de técnicas estadísticas. Los equipos de ventas son evaluados con base en tablas de frecuencia. Los resultados de estas evaluaciones son convertidos en parámetros que determinan sus niveles de efectividad.

\section{Elementos y características que dinamizan el aprendizaje colaborativo}

Con el propósito que la construcción del conocimiento se lleve a cabo, es necesario el rol del estudiante y su capacidad de identificar errores y dificultades que se generan durante este proceso. Para ayudar a los estudiantes en sus aprendizajes se ha implementado estrategias de gestión en el aula formando grupos de trabajo colaborativos incentivando la colaboración entre compañeros con el propósito de compartir, conocer y ampliar información sobre un tema en específico en este sentido, el aprendizaje colaborativo se considera como un aprendizaje consensuado mediante la cooperación de los miembros del grupo (Panitz, 1998).

Para Johnson (1999) lo más importante en la formación de grupos de trabajo colaborativo es supervisar que los elementos básicos estén claramente estructurados en cada sesión de trabajo. En este contexto, los elementos esenciales que contribuyen a que el aprendizaje colaborativo tenga éxitos son:

1. La responsabilidad individual: los estudiantes de forma individual son responsables de la parte de la actividad y tarea que les corresponde y han sido asignadas, para ellos es necesario tener claro las metas y ser perspicaces en medir su propio progreso tanto individual como grupal

2. La comunicación cara a cara: los estudiantes entre los equipos de trabajo intercambien información y se retroalimentan para mejorar su desempeño y obtener resultados de mayor calidad. A través de esta modalidad, los estudiantes se comprometen con sus pares y con las metas del grupo al que pertenecen. 
3. El trabajo en equipo: los estudiantes deben poseer habilidades personales y grupales además de contar con el conocimiento previos para resolver problemas en materia de estudio. En tal circunstancia, el trabajo en grupo contribuye al desarrollo de esas habilidades para resolver de forma conjunta los problemas planteados e ir desarrollando competencias de liderazgo, comunicación, confianza toma de decisiones y solución de conflictos.

4. El proceso de grupo: los integrantes establecen sus metas de forma periódica y deben evaluar sus actividades, con el propósito de identificar los cambios a realizarse para mejorar el desempeño del grupo y sus relaciones interpersonales.

Por otro lado, Johnson (1999) plantea que las características que se debe poseerlos aprendizajes colaborativos durante la construcción del conocimiento, algunas de ellas son:

1. La interactividad: no pude haber aprendizaje sino se produce la interacción entre dos o más estudiantes, mediando por un intercambio de opiniones y puntos de vista, depende de manera significativa la interacción que se origina en el proceso cognitivo y del aprendizaje de su par. En este contexto, se construye el conocimiento de la reflexión común, del intercambio de ideas y opiniones y más aun de un tema en común, de los cual, se obtiene un resultado de calidad.

2. La sincronía de la interacción: consiste en el diálogo orientado a realizar alguna actividad de manera conjunta. No se puede producir conocimiento sin respuesta inmediatas originado un diálogo espontáneo que generen la producción de nuevas ideas y respuestas. La colaboración es una actividad coordinada y sincrónica, que surge como resultado de un intento continuo por construir y mantener una concepción compartida de un problema. Sin embargo, el construir el conocimiento no es solamente un proceso social, tiene carácter individual de reflexión, es así, que sincronía la que defienden algunos teóricos al referirse a la colaboración afirmando que es "una actividad coordinada y sincrónica, que surge como resultado de un intento continuo por construir y mantener una concepción compartida de un problema".

3. La negociación: es un proceso en las que dos o más personas intentan obtener consentimiento y generara acuerdos en relación a una idea, tarea o problema. Según Dillenbourg, Baker, Blaye \& OMalley (1996) la negociación solo puede ocurrir si hay un espacio para que ocurra. Sin negociación el diálogo se transforma en un monólogo, es por ello que, en la interacción colaborativa, el individuo no debe imponer su visión, por el contrario.

En síntesis, según lo planteado por Johnson (1999) el aprendizaje colaborativo aumenta la seguridad en sí mismo, desarrolla el pensamiento crítico, fomenta la comunicación efectiva, propicia espacios de diálogo donde predomina el respeto mutuo, promueve el trabajo en equipo y disminuye el individualismo.

\section{El mecanismo de trabajar con el aprendizaje colaborativo en la estadística}

El aprendizaje colaborativo es un proceso de cambio cultural, donde los profesores son agentes del cambio en el espacio académico al facilitar que los estudiantes aprendan de esta forma. El aprendizaje colaborativo evita que los estudiantes dependan del profesor como 
figura de autoridad o experto en la materia a tratar en los procesos grupales. Tampoco le corresponde supervisar el aprendizaje del grupo, sino que su rol es ser un miembro más del grupo, estando al mismo nivel que los estudiantes, en el proceso de la construcción de conocimiento.

Según se plasma en el documento propuesto por el Tecnológico Monterrey (s.f.), para que la estrategia didáctica tenga éxito es necesario trasladar su concepto al aula, desempleado un papel vital el docente en preparar el ambiente propicio y trasmitir las instrucciones necesarias para la construcción del conocimiento a través del $\mathrm{AC}$, el cual incluirá lo siguiente:

1. Seleccionar el tema: el docente selecciona el espacio para comenzar con la colaboración, es recomendable iniciar con una lección para que los estudiantes se vayan adaptando al nuevo esquema o estrategia didáctica de aprendizaje. Para empezar con el proceso se sugiere en primer momento seleccionar el tamaño del grupo colaborativo y de la complejidad del tema a abordarse. Además, depende de los recursos, del tiempo y la naturaleza de la tarea.

En segunda instancia en necesario asignar a los estudiantes a los equipos, se sugiere formar grupos heterogéneos, resulta que este tipo de grupos son más fuertes debido que se generan discusiones, explicación, fundamentación sobre la temática tratada.

En tercera instancia es importante el arreglo o rediseño de los muebles del aula, de manera que facilite la movilidad del docente y los equipos quienes deben estar cerca uno de otros y frente a frente para el intercambio de ideas y materiales de trabajo.

2. Roles: además, para que la técnica resulte positiva, es necesario la asignación de roles dentro de cada equipo conformado, entre los sugeridos son los siguientes:

a. Supervisor es quien monitorea a los miembros de los equipos, lleva el consenso y detiene el trabajo cuando algún integrante desea aclarar dudas, su rol es similar a un modelador.

b. Motivador es quien se asegura que todos los integrantes del grupo tengan la oportunidad de participar en el trabajo y elogia a los participantes por sus contribuciones.

c. Administrador de materiales su rol es organizar y proveer de material suficiente para la realización de tareas o proyectos

d. Observador es quien debe monitorear el comportamiento del grupo acordada previamente, este emite las observaciones al docente.

e. Secretario es quien debe tomar las notas e ideas generadas en el grupo con la finalidad de consolidar, asegurando que la información sea clara para todos y da lectura socializando el producto final con el propósito de retroalimentar el tema tratado.

f. Controlador de tiempo su rol es controlar el tiempo y controlar que el equipo realice las actividades de acuerdo a estándares establecidos previamente para terminar a tiempo las tareas. 
3. Evaluación: la evaluación debe ocurrir a nivel individual como dentro de los grupos, la asignación de roles sirve como un mecanismo formal para evaluar el progreso en grupo, es importante socializar, describir y detallar a los estudiantes la manera de evaluar las actividades colaborativas a ser valoradas. Al igual que otro método o técnica de enseñanza, el sistema de evaluación está desarrollado tanto formativa y sumativa y pueden aplicarse en varias etapas del proceso colaborativo ya sea por el docente, el mismo estudiante o por sus compañeros de grupo. En este sentido, en la evaluación formativa las actividades son usadas para proveer con retroalimentación, evaluando para motivar a los estudiantes a alcanzar niveles más altos de desempeño, mientras que la evaluación sumativa las actividades son usadas para juzgar el término de productos finales, la competencia y que se demuestre mejora.

4. Recursos: es responsabilidad de la institución, proveer con los recursos necesarios para el trabajo con el aprendizaje colaborativo y este pueda llevarse a cabo de la mejor manera, estos recursos se enumeran a continuación:
a. Espacios
b. Bibliotecas
c. Recursos tecnológicos

\section{El aprendizaje colaborativo: un enfoque social}

La inserción del aprendizaje colaborativo en los estudiantes del Instituto Superior Tecnológico ha potenciado a mejorar las relaciones interpersonales, involucrar al estudiante hacia su propio aprendizaje fomentando el sentido de pertenencia y autoestima, surgen espacios para la retroalimentación. El aprendizaje colaborativo tiene su fundamento teórico en el constructivismo social como lo señalan (Barkley, Major \& Cross, 2008; Barros \& Verdejo, 2001; Maldonado (2008); entre otros, cuyo enfoque se fundamenta en la Sociología, siendo Shütz (padre del constructivismo social), quien afirma que la realidad social es una construcción humana dependiente de la interacción social, que genera significados y los transmite a través de la cultura (Saso, Aiguadé, Gallart \& Carol, 2003).

Adicionalmente, es necesario mencionar que el aprendizaje colaborativo está ligado al constructivismo, donde las personas son quienes construyen sus propios conocimientos a través de la experiencia y la relación con el ambiente, postulado de su principal exponente Jean Piaget, quien expresa que esta modalidad de aprendizaje consiste en la generación de estructuras cognoscitivas que se crean a través de la modificación de los reflejos iniciales del recién nacido y que se van enriqueciendo a través de la interacción del individuo con el medio (Gómez, 2000).

\section{METODOLOGÍA}

La metodología utilizada es de tipo exploratorio, bibliográfica de campo y cuantitativa. Exploratorio porque se ejecutó un análisis en relación a la estrategia metodológica el Aprendizaje Colaborativo que los docentes utilizan en la construcción del conocimiento en el Instituto Superior Tecnológico Guaranda, además es de carácter bibliográfica porque se realizó 
la revisión del tema en libros para conocer el estado de la cuestión, mediante la búsqueda, recopilación, estimación, crítica e información bibliográfica sobre temas relacionadas con la presente investigación, también se utilizó el estudio de campo mediante encuestas que se realizó a estudiantes del instituto y mediante el método cuantitativo se recopiló la información para tabular y posteriormente presentar los resultados obtenidos en el trabajo de investigación.

\section{RESULTADOS}

Respecto al aprendizaje colaborativo en la construcción del conocimiento, la información obtenida de las encuestas aplicadas a estudiantes, con relación a las preguntas planteadas, arrojó los siguientes resultados:

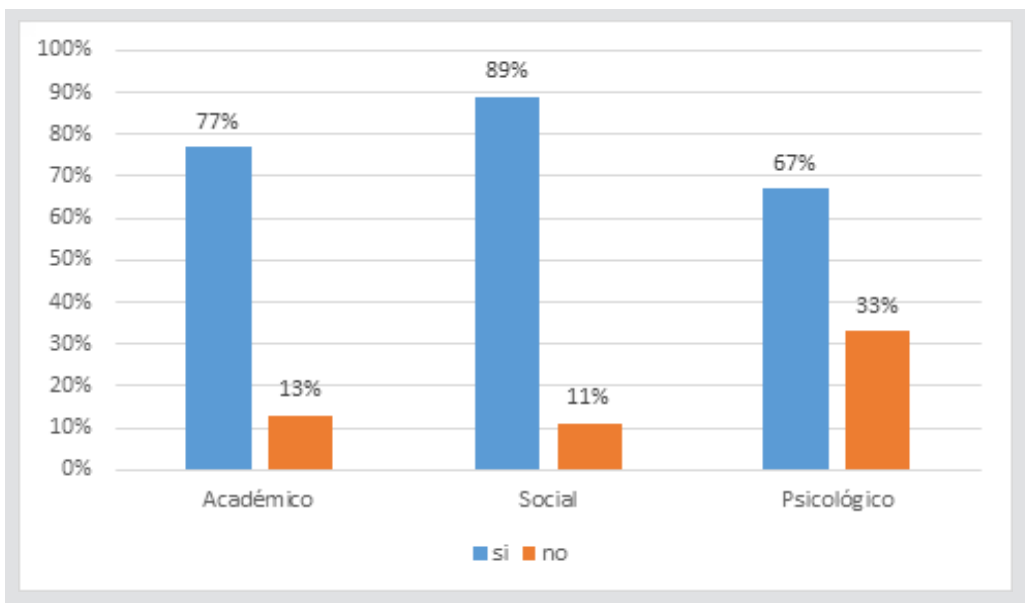

Figura 1. Porcentaje de docentes y estudiantes que consideran que el aprendizaje colaborativo general beneficios de tipo académico, social y psicológicos

De acuerdo a la figura 1, los beneficios que aporta el aprendizaje colaborativo en la estadística, es al aspecto social y académico con un $89 \%$ y $77 \%$ respectivamente, mientras otro grupo de encuestados señalan que se ven beneficiados en el aspecto psicológico.

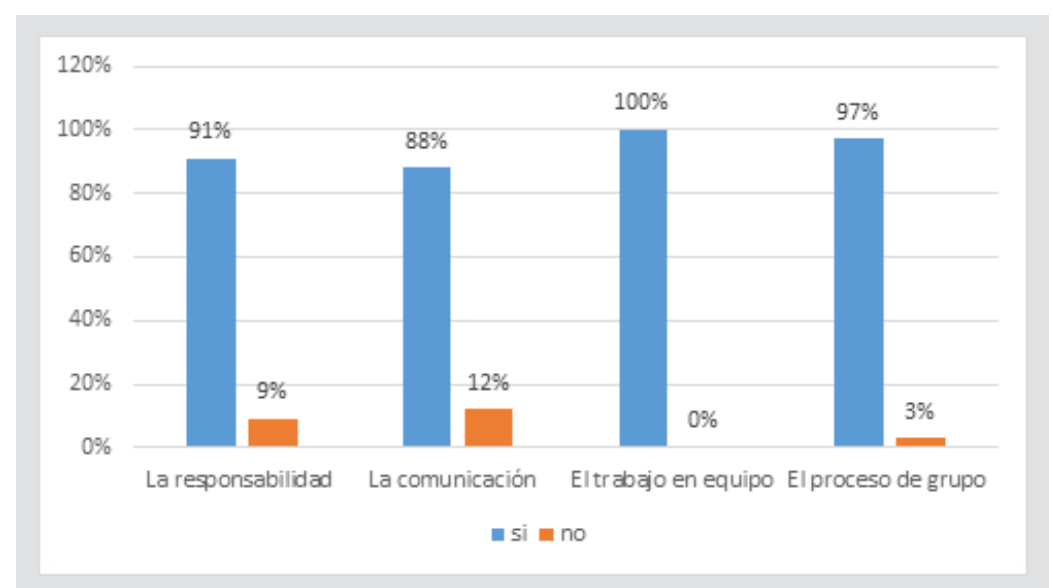

Figura 2. Porcentaje de docentes y estudiantes que consideran que el aprendizaje colaborativo sí fomenta la responsabilidad, la comunicación, el trabajo en equipo y el proceso de grupo. 
En referencia a la figura 2, los encuetados expresan que el aprendizaje colaborativo fomenta el trabajo en equipo con el $100 \%$, el proceso de grupo con el $97 \%$, la responsabilidad en un $91 \%$, mientras en un porcentaje menor con el $88 \%$ responden que esta estrategia de enseñanza fomenta la comunicación.

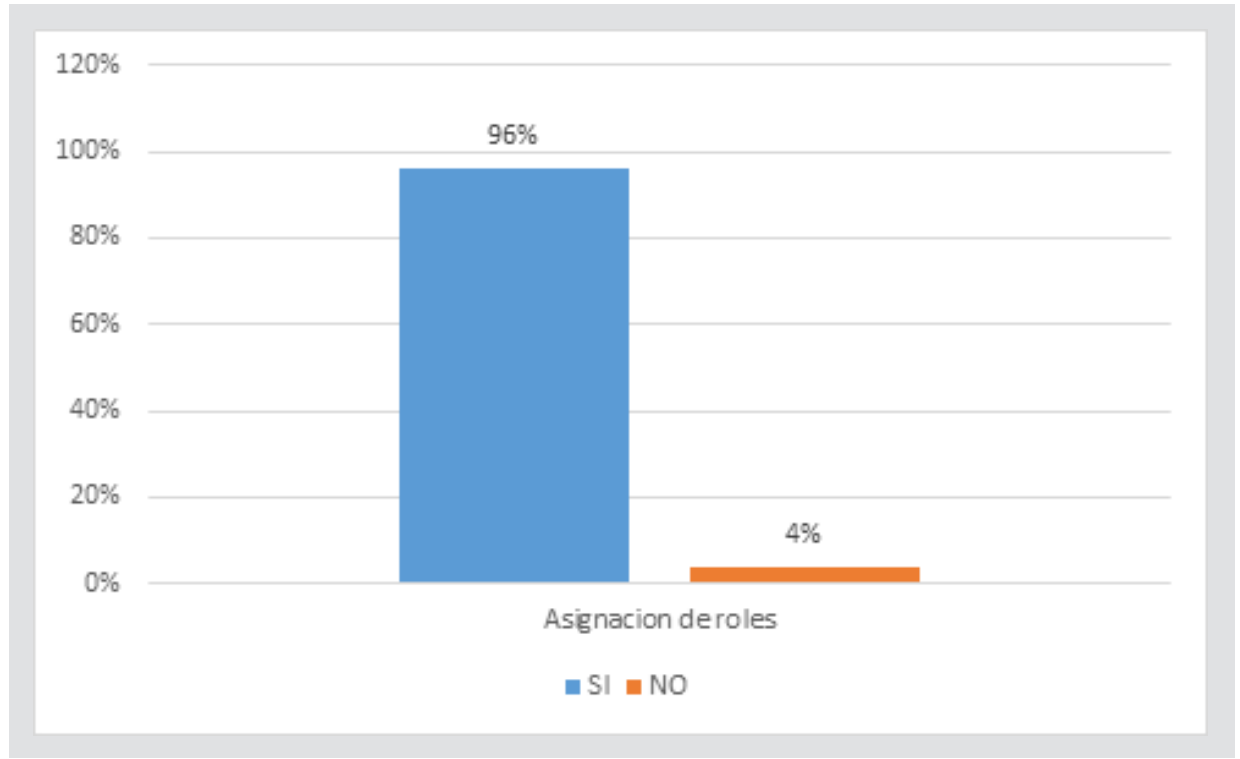

Figura 3. Porcentaje de docentes y estudiantes que consideran que la asignación de roles en el aprendizaje de la estadística promueve el desarrollo social

De acuerdo a la figura 3, el 96\% de encuestados consideran que asignación de roles en el aprendizaje colaborativo facilitan y promueven a desarrollarse socialmente. Mientras que un $4 \%$ manifiesta que no influyen en desarrollares socialmente.

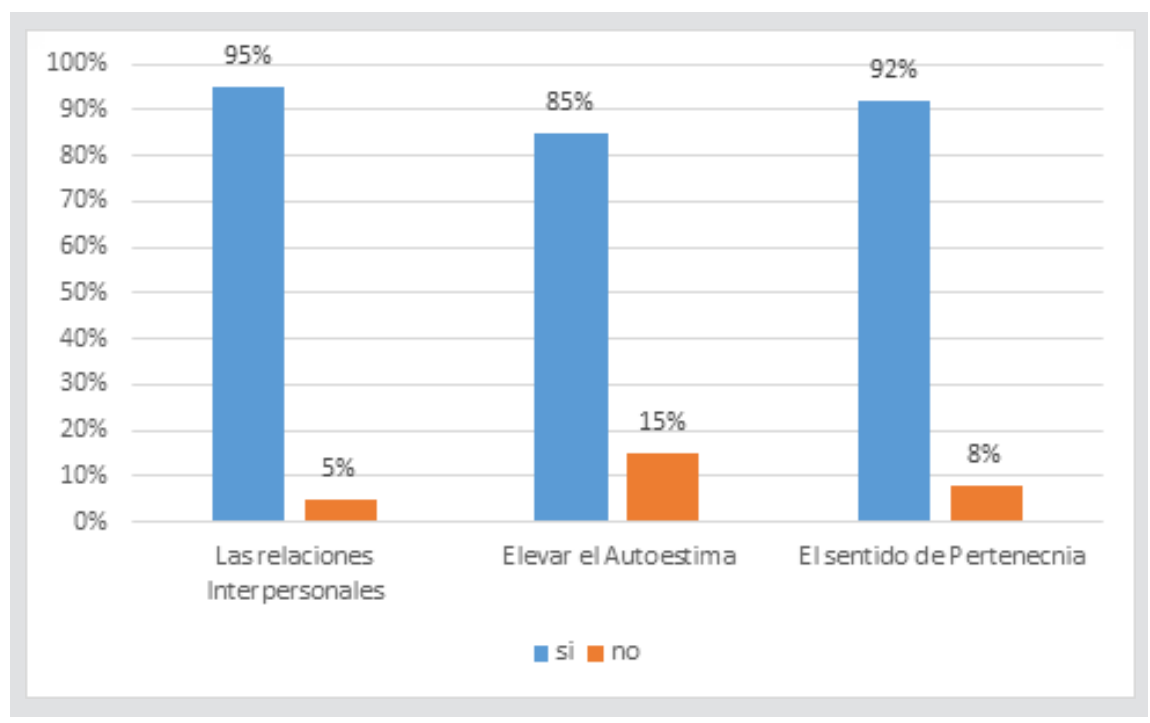

Figura 4. Porcentaje de docentes y estudiantes que consideran que el aprendizaje colaborativo sí fomenta las relaciones interpersonales, elevan la autoestima y el sentido de pertenencia. 
De acuerdo con la figura 4 se identificó que la estadística y el aprendizaje colaborativo, como enfoque social, el 95\% de los encuestados afirman que sí mejora significativamente las relaciones interpersonales, además de mejorar el sentido de pertenencia y elevar la autoestima en los estudiantes. Esto refleja que la metodología contribuye socialmente a formar profesionales democráticos y que trabajen de forma conjunta.

\section{DISCUSIÓN}

La estadística descriptiva e inferencial en relación a la investigación realizada por Maldonado (2008) arroja que "el propósito es de crear situaciones en las cuales se generen interacciones productivas entre los estudiantes; el empleo de esa estrategia de aprendizaje exige a los estudiantes tomar decisiones, proponer soluciones, negociar ideas y construir la propuesta. Al mismo tiempo, promueve la adquisición de destrezas sociales colaborativas. En referencia a lo antes expuesto se puede manifestar que el aprendizaje colaborativo como influencia social, promueve el trabajo en equipo la responsabilidad y la comunicación mediante la asignación de roles en la construcción del conocimiento durante en el proceso de enseñanza, además de mejorar el sentido de pertenencia, las relaciones interpersonales y la autoestima; se constituye en un método educativo como hecho social que aporta al estudiante la posibilidad de desenvolverse de forma activa y su participación sea proactiva en el aula y la sociedad. Estas afirmaciones también coinciden con los estudios realizados por Morales, Pineda, \& Saucedo (2015, p. 183) en el cual se destaca "el respecto a la práctica docente, ...el aprendizaje colaborativo, la educación inclusiva y ...el respecto a las posibilidades de cambio.

\section{CONCLUSIONES}

La estadística por medio del aprendizaje colaborativo como influencia social tiene su fundamento teórico en el constructivismo social que ayuda a mejorar de forma notable el sentido de pertenencia, el sentido crítico y elevar la autoestima para desenvolver de mejor manera en el aula y la sociedad

El aprendizaje colaborativo transforma la manera de enseñar la estadística, promueve el trabajo colaborativo y el trabajo en equipo, la responsabilidad, la comunicación efectiva. con la intencionalidad de generar la interacción entre dos o más estudiantes, mediando por un intercambio de opiniones y puntos de vista de los cual, se obtiene un aprendizaje de calidad

En el proceso del aprendizaje colaborativo la clave del éxito es la asignación de roles para poder interpretar un ejercicio estadístico, para organizar los espacios de aprendizaje, los grupos de trabajo y guiar en la construcción del conocimiento.

El aprendizaje colaborativo beneficia sustancialmente en el rendimiento académico, aporta al desarrollo social del estudiante y mantiene motivado al alumno durante el proceso de enseñanza lo que mejora su estado emocional. 


\section{REFERENCIAS}

Barkley, E., Major, C. H., \& Cross, K. (2008). Técnicas de aprendizaje colaborativo. Manual para el profesorado universitario.

Barros, B., \& Verdejo, M. F. (2001). Entornos para la realización de actividades de aprendizaje colaborativo a distancia. Inteligencia Artificial. Revista Iberoamericana de Inteligencia Artificial, 5(12), 39-49. https://www.redalyc.org/pdf/925/92551205. pdf

Dillenbourg, P., Baker, M., Blaye, A., \& OMalley, C. (1996). The evolution of research on collaborative learning. Elsevier.

Gómez,L.F.(2000).Delateoríageneralalaenseñanza en elaula.Sinéctica, RevistaElectrónica de Educación, (17), 39-51. https://www.redalyc.org/pdf/998/99817933006.pdf

Johnson, D. J. (1999). Los nuevos circulos de aprendizaje. Aique.

Maldonado, M. (2008). Aprendizaje basado en proyectos colaborativos. Una experiencia en educación superior. Laurus revista de Educación, 14(28), 158-180.

Morales, M.G. V.,Pineda,J.M.M., \&Saucedo, F.M.(2017).Educacióninclusivay aprendizaje colaborativo en el aula: un estudio de la práctica docente universitaria. Revista de Educación Inclusiva, 8(3). http://www.revistaeducacioninclusiva.es/index.php/REI/ article/download/97/94

Panitz, T. A. (1998). Encouraging the Use of Collaborative Learning in Higher Education. Garland Publishing.

Roberts, T. S. (2005). Computer-supported collaborative learning in higher education: An introduction. Idean Group Publishing.

Saso, C. E., Aiguadé, I. P., Gallart, M. S., \& Carol, M. R. V. (2003). Comunidades de aprendizaje: transformar la educación (Vol. 177). Graó.

Tecnológico Monterrey. (s.f.). Aprendizaje Colaborativo. http://www.itesca.edu.mx/ documentos/desarrollo_academico/metodo_aprendizaje_colaborativo.pdf 